\section{RMD Open}

Rheumatic \& Musculoskeletal Diseases
To cite: Westerlind $\mathrm{H}$, Delcoigne B, Askling J. Siblings of patients with rheumatoid arthritis have an increased mortality rate: a Swedish cohort study. RMD Open 2020;6: e001197. doi:10.1136/ rmdopen-2020-001197

- Additional material is published online only. To view please visit the journal online (http://dx.doi.org/10.1136/rmdo pen-2020-001197)

Received 7 February 2020 Revised 27 March 2020 Accepted 19 April 2020
Check for updates

(C) Author(s) (or their employer(s)) 2020. Re-use permitted under CC BY-NC. No commercial re-use. See rights and permissions. Published by BMJ.

Department of Medicine, Solna, Karolinska Institutet, Stockholm, Sweden

Correspondence to Helga Westerlind; Helga.Wester lind@ki.se

\title{
Siblings of patients with rheumatoid arthritis have an increased mortality rate: a Swedish cohort study
}

\author{
Helga Westerlind (D), Bénédicte Delcoigne (D), Johan Askling (D)
}

\section{ABSTRACT}

Objectives To estimate the mortality among siblings of patients with rheumatoid arthritis (RA) and put any excess mortality among these in relation to the mortality among patients with RA.

Methods Using prospective nation-wide registers, we identified patients diagnosed with new-onset RA 2001-2017 $(n=8137)$, patients with prevalent $R A$ 2006-2017 ( $n=25$ 464), matched general population comparator subjects to all RA patients ( $n=22$ 457/68 674) and full-siblings of all groups ( $n=28$ 878/91546).

We followed all cohorts until death, 31 December 2018, migration and (for non-RA subjects) RA diagnosis. We compared patients with RA versus the general population, and siblings of RA versus siblings of the general population using Cox regression, including adjustment for socio-economy. Results The HR of death versus the general population was $1.11(95 \% \mathrm{Cl} 1.01$ to 1.22$)$ for incident and $1.46(95 \% \mathrm{Cl}$ 1.39 to 1.52) for prevalent patients with RA. The siblings of these patient groups were also at increased risk of death ( $\mathrm{HR}=1.10,95 \% \mathrm{Cl} 1.01$ to 1.20 and $1.09,95 \% \mathrm{Cl} 1.04$ to 1.13 , respectively), with little impact of adjustment for socio-economy.

Conclusion The mortality in RA is increased, but around one-fifth of this excess is present also among their siblings. Previous literature using general population rates for comparison has thus likely overestimated the direct impact on mortality attributable to RA. To bring down excess mortality in RA, optimal disease control is important but may not suffice.

\section{INTRODUCTION}

Rheumatoid arthritis (RA) is a chronic, autoimmune disease characterised by joint inflammation and joint destruction. ${ }^{1}$ Compared with the general population, patients with RA have an increased mortality rate. ${ }^{2-4}$ This increase has been attributed to consequences of the RA disease, such as inflammation and comorbidities. ${ }^{3}$ During recent time periods, presumably as a result of better treatments and improved RA disease control, the mortality in RA has decreased, yet it has remained elevated compared with the general population. ${ }^{2} 45$

\section{Key messages}

What is already known on this subject

- The mortality rate among patients with RA is higher than in the general population; this increase is detectable already $5-10$ years after the RA diagnosis.

What this study adds

- The increased mortality rate among the patients with RA extends, although at a lower magnitude, also to their full siblings. This risk increase cannot be readily explained by socio-economic status.

- Our findings suggest that previous mortality estimates from cohorts of patients with RA versus the general population are likely to have over-estimated the direct contribution of RA to the mortality.

How might this impact on clinical practice or future developments

- To bring down the excess mortality rate among patients with RA, optimised RA disease control is important, but may not be enough.

In most previous studies on RA mortality, patients have been compared with the general population or to healthy controls, ${ }^{2-4}$ and the difference in mortality has served as a measure of the excess mortality risk attributable to RA. For one of the major co-morbidities in RA, cardiovascular disease, we recently demonstrated that an increased risk of acute coronary syndrome was not restricted to patients with RA but was present, although at a lower level of excess risk, also among their siblings (who have similar genetic and lifestyle set-up) ${ }^{6}$ These findings suggest, besides a cause-and-effect association between RA and cardiovascular disease, also the existence of shared risk factors for RA and coronary disease.

In this present project, we set out to investigate whether, and if so, how much of the increased overall mortality among patients with RA that might be explained by factors present already before RA diagnosis, using sibling risks as a means to assess the counterfactual ideal of 
comparing patients with RA to (individuals similar to) themselves in the absence of RA.

\section{METHODS}

\section{Study setting and design}

We used Swedish nation-wide and population-based registers based on prospectively collected data to perform a cohort study of the relative mortality among patients with RA and their siblings. The registers and linkages have been described elsewhere ${ }^{7}$ (see also online supple mentary file for details). Ethical permission was obtained from the Stockholm ethics review board (2015/1844$31 / 2)$.

\section{Identification of patients with RA}

Through the Swedish Rheumatology Quality Register (SRQ), we identified a cohort of patients with incident RA, born 1932 or later, and diagnosed with RA 2001-2017 within 1 year of RA symptom onset. RA sero-status was based on rheumatoid factor (RF) status as defined by the reporting clinician. Based on the National Patient Register (NPR), we identified two additional (overlapping) RA cohorts, one of incident RA diagnosed 2006-2017, and one with prevalent RA during the same period, using previously devised algorithms. ${ }^{8}$ For the NPR cohorts, seropositivity was for the prevalent defined as having a main diagnosis of seropositive RA (International Classification of Diseases (ICD), Tenth Revision=M05) in at least half of the visits with a main diagnosis code of RA during the study period, and for the incident NPR cohort, both visits were required to have seropositive RA as the main diagnosis.

\section{Matched general population comparator subjects and relatives} For each unique patient with RA, we randomly selected up to five general population comparator subjects matched on age, sex and residential area, from the Swedish population register. Using the Swedish multigeneration register (MGR), we then identified all fullsiblings, born within a 5-year interval, of all unique patients with RA and their comparator subjects.

\section{Follow-up and outcome}

Comparator subjects and siblings were required to be alive and residing in Sweden at the time of the diagnosis of the index patient, which defined the start of follow-up. We followed all individuals up until emigration, death, 31 December 2018 and (non-RA subjects only) any diagnosis of RA.

\section{Statistical analysis}

We calculated mortality rates for each cohort, and age- and sex-standardised mortality rates against the age and sex distribution among the incident cases with RA identified through SRQ using the R-package dsr. We then used Cox proportional hazards models to estimate the HR of death, comparing patients with RA to their general population comparator subjects, and siblings of the patients with RA to the siblings of their general population comparator subjects.
We adjusted for age, sex and calendar period of the index patient's RA diagnosis. A robust sandwich estimator was used for calculating the 95\% CIs to account for dependencies within the data. The proportional hazards assumption was tested with the R function cox.zph.

\section{Exploratory analysis}

To further investigate the findings, we explored the impact of socio-economic status by adjusting our analysis for income the year before the start of follow-up (adjusted for inflation and divided into deciles), and educational level and marital status by the time of diagnosis. We also calculated the sexspecific mortality rates and HRs for each group and looked at the distribution of the ICD chapters among the reported causes of death in the sibling groups.

\section{Role of the funding source}

This work was supported by the Swedish Research Council, Stockholm County Council (ALF), the Heart Lung Foundation, Karolinska Institutet (Strategic Research Area Epidemiology), The Nordic Research Council (Nordforsk) and the Rheumatology Research Foundation (FOREUM). Funders had no impact on the design or interpretation of the study or its results.

\section{RESULTS}

In SRQ, we identified 17363 patients of which 10588 had as least one sibling in MGR. After filtering out the sibling pairs born more than 5 years apart (removing $\mathrm{N}=9924$ pairs, median difference in age between the siblings filtered out was 8 years), and matching to general population comparator subjects on age, sex and residential area, we ended up with 8137 patients with new-onset RA with 10261 siblings, and 22457 individually matched general population comparator subjects on age, sex, calendar year of entry and residential area, with 28878 full-siblings after applying the same filtering criteria. The median age at the start of follow-up was 55 years and the median follow-up time was 7 years (table 1 ).

Via NPR, we identified 25464 prevalent patients with RA who had 32597 siblings, and 68674 general comparator subjects with 91546 siblings. The median age at the start of follow-up was 56 years and the average follow-up time 9 years (table 2 ).

Through NPR, we additionally identified 10708 incident patients with RA with 13455 full-siblings and 29264 matched comparator subjects with 38070 siblings. The median age at the start of follow-up was 56 years and the average follow-up time 6 years (online supplementary table S1).

\section{Mortality among patients with RA}

In the incident SRQ cohort, we found 608 deaths among the cases and 1397 among the general population comparator subjects; the sex- and age-standardised mortality rates were 9.9 and 8.9 per 1000 person-years, respectively. 


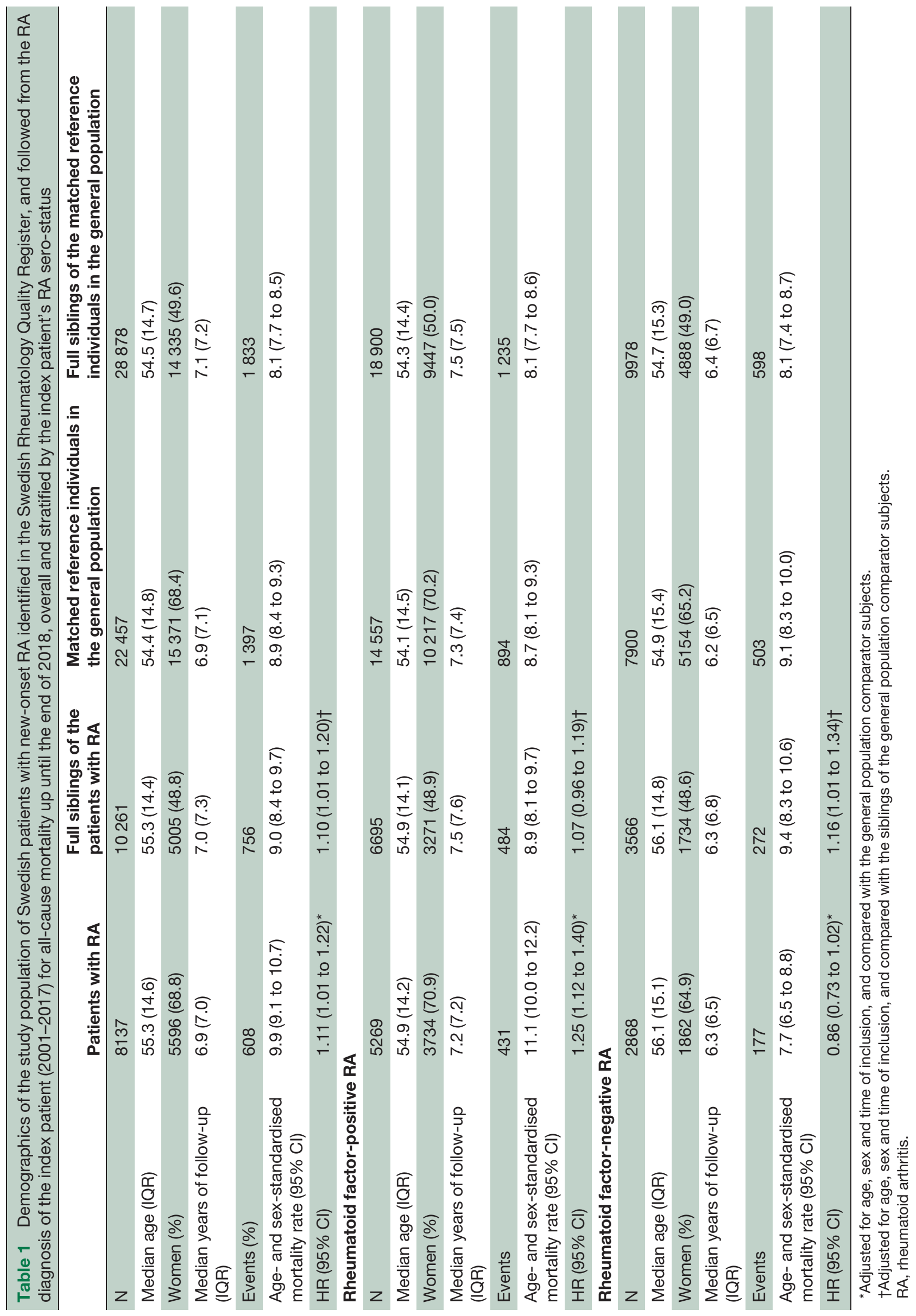




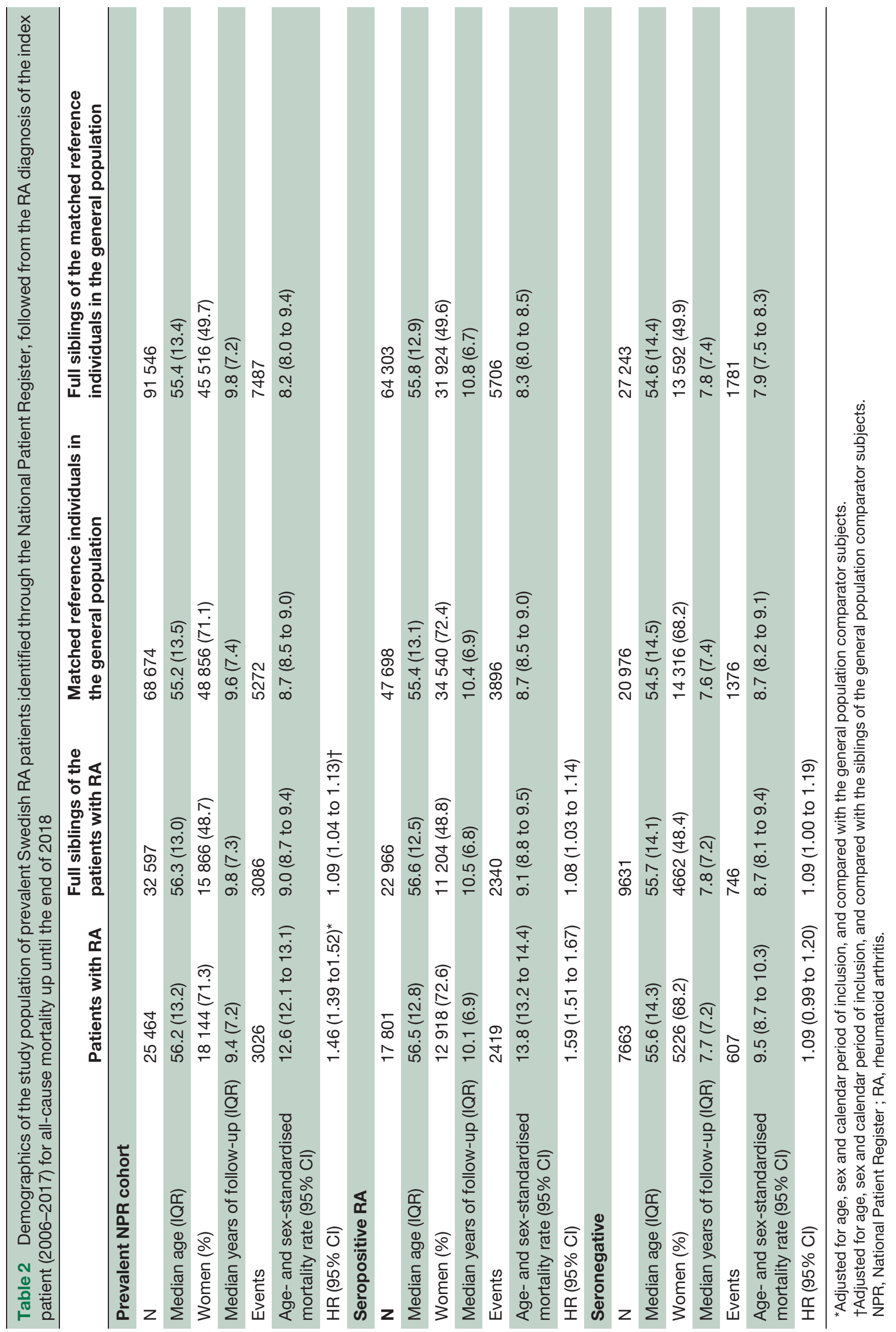


This resulted in an overall HR for mortality of $1.11(95 \%$ CI 1.02 to 1.22), which increased from 0.59 (95\% CI 0.41 to 0.84 ) the year after RA diagnosis to 1.55 (95\% CI 1.29 to 1.94 ) 10 or more years after diagnosis (online supple mentary table S2). Kaplan-Meier curves can be found in online supplementary figure S1. Formal testing of the Cox proportional hazards assumption showed that the proportional hazards assumption did not hold ( $\mathrm{p}$ global=9.0e-4), as indicated by the follow-up time-specific HRs presented in online supplementary table S2.

Among the prevalent patients, the mortality rates were 12.6 for the population with RA and 8.7 for their general population comparator subjects, HR=1.46 (95\% CI 1.39 to 1.52) (table 2).

For the incident patients identified via NPR, the corresponding overall HR was 1.19 (95\% CI 1.09 to 1.29 ) (online supplementary table S1).

\section{Mortality among the siblings}

For the siblings of the incident SRQ cohort, the HR of death versus the siblings of the general population subjects was 1.10 (95\% CI 1.01 to 1.20) Kaplan-Meier curves can be found in online supplementary figure S2. For the two other RA sibling groups, we observed very similar HRs: 1.09 (95\% CI 1.04 to 1.13) for siblings of the prevalent RA cohort (table 2) versus the siblings of their general population controls, and 1.08 (95\% CI 1.00 to 1.16) for siblings of the incident RA cohort (online sup plementary table S1). These HRs for siblings remained stable over time since the start of follow-up (online sup plementary table S2). Formal testing of the Cox proportionality assumption was not rejected ( $\mathrm{p}$ global $=0.65$ ).

\section{Sensitivity analyses}

When we stratified the RA-cohort defined via SRQ by RF status, the HR for patients with seropositive RA was 1.25 (95\% CI 1.12 to 1.40 ), and 0.86 (95\% CI 0.73 to 1.02 ) for seronegative RA. The HR of death for siblings of patients with seropositive RA $(1.07,95 \%$ CI 0.96 to 1.19$)$ was similar to that for siblings of patients with seronegative RA (1.16 (95\% 1.01-1.34)); the interaction between RF and sibling status was not significant.

\section{Exploratory analysis}

Income, marital status and educational level were all strongly associated with mortality in the general population (online supplementary table S3). Including these variables in our full model had little impact on the HRs for patients and for their siblings, respectively (online supplementary table S4). Mortality rates for all four groups in the incident SRQ cohort, stratified by sex-and age-standardised mortality rates against the incident SRQ cohort are presented in online supplementary table S5 together with HRs stratified by sex. The HRs showed a slightly higher mortality increase among female patients with RA versus their matched female general population comparator subjects than among male patients with RA (1.13 (95\% CI 0.99 to 1.29) among women and 1.09 (95\%
CI 0.95 to 1.26 ) among males), and among sisters of patients with RA versus sisters of the comparator population than among brothers of patients with RA (HR among sisters $=1.21(95 \%$ CI 1.06 to 1.38$)$ versus HR for brothers $=1.02(95 \%$ CI 0.91 to 1.15$)$ ). These patterns also held true when adjusting for income, educational level and marital status (online supplementary table S4).

The distribution of the causes of deaths did not differ between the sibling groups (online supplementary table S6).

\section{DISCUSSION}

This study is, to our knowledge, the first to seek to partition the effects of RA from the effects of its context, on mortality. We performed a sibling analysis to investigate if, and how much of, the increased risk of premature mortality among patients with RA that might be attributed to factors other than the RA disease itself. Among the siblings of patients with RA, we found that the mortality was $10 \%$ higher compared with siblings of the general population comparator subjects, which in turn was largely similar to the excess mortality among the patients themselves during the first few years after their RA diagnosis.

In our unselected cohort of RA of all durations, we observed, as expected, a higher excess mortality (50\%) compared with the general population, but similar to the findings in the incident RA cohorts, a 10\% increase in mortality among their full-siblings. Comparing these absolute rate differences to each other, as much as around onefifth of the excess mortality observed in prevalent patients with RA may be attributable to other factors, enriched in these individuals, then the RA disease PER SE.

For the age-adjusted mortality rates stratified on sex, we did, as expected, observe a higher mortality rate among men compared with women. The HRs demonstrated a slightly higher level of risk increase among females (female patients vs female general population, and sisters of RA patients vs sisters of population controls) than among males (male patients vs male comparator subjects, and brothers of RA patients vs brothers of the population controls). However, these results need to be interpreted with caution: There are important (and to some extent also age-dependent) differences in the RA subtype distribution and phenotype among males and females. Thus, before concluding that any sex-differences exist, a more extensive analysis, involving also stratification on index patient's sex, and the use of same-sex pairs, would be needed, something this study was not designed for.

Similar to other inception cohort studies of RA, we noted a reduced mortality in patients with RA during the first year of follow-up. We interpret this as a result of diagnostic intensity bias (individuals with a projected survival less than 1 year but who develop RA are less likely to have it diagnosed, let alone entered into longitudinal monitoring systems such as a clinical quality register). When testing the proportionality assumption in the statistical model, and because of the increasing mortality HR among patients 
counting from RA diagnosis through follow-up, the proportional hazards assumption did not hold for the overall HR for patients; follow-up time-specific HRs are presented in online supplementary table S2. These are in keeping with data from previous studies. ${ }^{2}$ Importantly, for the group of interest, namely the siblings for whom the modelling of mortality risk was fitted separately from that of the patients, the proportional hazards assumption was not violated.

Our present report extends on a previous study on mortality $^{2}$ in which we demonstrated that the mortality among patients with RA is decreasing in absolute numbers, but, as this decrease is happening in the general population as well, the risk difference remains. Taking the risk increase among the siblings into account, our results indicate that patients with RA might be a group of individuals who also in the absence of RA might be at higher risk of premature mortality. This implies that better control of the RA disease might not be sufficient to bring down the mortality rate to that of the general population.

The aim of this project was to assess if the RA disease itself is attributable to the increased mortality rate among the patients with RA or if there could be other factors, present already before disease onset, contributing. When taking socio-economic factors into account, the $10 \%$ risk increase among the siblings remained, and the causes of deaths reported for the siblings showed almost identical distributions of the reported ICD chapters for all sibling groups. Further studies to better understand the reasons behind the risk increase among the siblings are needed. We and others have previously described a higher mortality in seropositive than in seronegative RA. ${ }^{29}{ }^{10}$ Interestingly, we observed no such subset difference in mortality among their siblings.

Limitations of our study include lack of individual-level data and the possibility to adjust for lifestyle factors such as smoking and diet. However, through our register setting, we had the possibility to adjust for socio-economic factors that were all strongly associated with mortality in our general population cohorts. Other strengths include nationwide, register-based setting with prospectively collected, virtually complete, follow-up data, and large-scale identification of siblings and their mortality, independent of the index individual's RA status. Because of the large numbers and internal consistency of the results, we consider the estimates robust.

In conclusion, siblings of patients with RA seem to be at an increased risk of premature death. This finding implies that previous mortality estimates in RA comparing patients to the general population or healthy controls may have overestimated the increased mortality risk attributable to the RA disease itself by some $20 \%$. Clinically, our results point to the enrichment of additional risk factors for death among patients with RA (and their siblings).

Contributors HW had full access to all of the data used for the analysis in this study and takes full responsibility for the integrity of the data and the accuracy of the data analysis. Study concept and design: JA, HW. Acquisition of data: JA, HW. Statistical analysis: HW, JA, BD. Analysis and interpretation of data: all authors. Drafting of manuscript: HW, JA. Critical revision of manuscript and final approval given: all authors. Obtained funding: JA. Study supervision: JA.

Funding The authors have not declared a specific grant for this research from any funding agency in the public, commercial or not-for-profit sectors.

Competing interests Karolinska Institutet (JA as principal investigator) has or has had research agreements with Abbvie, Astra-Zeneca, BMS, Eli Lilly, MSD, Pfizer, Roche, Samsung Bioepis, Sanofi and UCB, mainly in the context of safety monitoring of biologics via ARTIS/Swedish Biologics Register.

Patient consent Waived by the Region Stockholm Ethics Review Board because of the register-based setting

Data sharing statement No data are available.

Provenance and peer review Not commissioned; externally peer reviewed.

Open access This is an open access article distributed in accordance with the Creative Commons Attribution Non Commercial (CC BY-NC 4.0) license, which permits others to distribute, remix, adapt, build upon this work non-commercially, and license their derivative works on different terms, provided the original work is properly cited, appropriate credit is given, any changes made indicated, and the use is non-commercial. See: http://creativecommons.org/licenses/by-nc/4.0/.

\section{ORCID iDs}

Helga Westerlind http://orcid.org/0000-0003-3380-5342

Bénédicte Delcoigne http://orcid.org/0000-0002-2716-5679

Johan Askling http://orcid.org/0000-0003-0433-0616

\section{REFERENCES}

1 Smolen JS, Aletaha D, Barton A, et al. Rheumatoid arthritis. Nat Rev Dis Primers 2018;8:18001.

2 Holmqvist M, Ljung L, Askling J. Mortality following new-onset rheumatoid arthritis: has modern rheumatology had an impact? Ann Rheum Dis 2018;77:85-91.

3 Myasoedova E, Davis JM 3rd, Crowson CS, et al. Epidemiology of rheumatoid arthritis: rheumatoid arthritis and mortality. Curr Rheumatol Rep 2010;12:379-85.

4 Provan SA, Lillegraven S, Sexton J, et al. Trends in all-cause and cardiovascular mortality in patients with incident rheumatoid arthritis: a 20-year follow-up matched case-cohort study. Rheumatology (Oxford) 2020;59:505-512.

5 Lacaille D, Avina-Zubieta JA, Sayre EC, et al. Improvement in 5 -year mortality in incident rheumatoid arthritis compared with the general population-closing the mortality gap. Ann Rheum Dis 2017;76:1057-63.

6 Westerlind H, Holmqvist M, Ljung L, et al. Siblings of patients with rheumatoid arthritis are at increased risk of acute coronary syndrome. Ann Rheum Dis 2019;78:683-687.

7 Askling J, Fored CM, Geborek P, et al. Swedish registers to examine drug safety and clinical issues in RA. Ann Rheum Dis 2006:65:707-12.

8 Waldenlind K, Eriksson JK, Grewin B, et al. Validation of the rheumatoid arthritis diagnosis in the Swedish National Patient Register: a cohort study from Stockholm County. BMC Musculoskelet Disord 2014;15:432.

9 Humphreys $\mathrm{JH}$, Warner A, Chipping J, et al. Mortality trends in patients with early rheumatoid arthritis over 20 years: results from the Norfolk Arthritis Register. Arthritis Care Res (Hoboken) 2014;66:1296-301.

10 Radovits BJ, Fransen J, Al Shamma S, et al. Excess mortality emerges after 10 years in an inception cohort of early rheumatoid arthritis. Arthritis Care Res (Hoboken) 2010;62:362-70. 